ISSN: 2386-3919 - e-ISSN: 2386-3927

DOI: https://doi.org/10.14201/et202038187109

\title{
VALORACIÓN DE LA FORMACIÓN RECIBIDA POR ESTUDIANTES DEL TÍTULO DE GRADO DE MAESTRO EN EDUCACIÓN PRIMARIA
}

\author{
Assessment of the Training Received by Students \\ of the Teacher's Degree in Primary Education
}

Cristo EsPARCIA JimÉNEZ* y Miguel PÉREZ FERRA***1

* Junta de Andalucía

Correo-e: cristo.esparcia.edu@juntadeandalucia.es

**Universidad de Jaén

Correo-e: mperez@ujaen.es

Recibido: 30/05/2020; Aceptado: 22/07/2020; Publicado: 30/12/2020

Ref. Bibl. CRISTO ESPARCIA JIMÉNEZ y MIGUEL PÉREZ FERRA. Valoración de la formación recibida por estudiantes del Título de Grado de Maestro en Educación Primaria. Enseñanza \& Teaching, 38, 1-2020, 87-109.

RESUMEN: La sociedad del conocimiento y la globalización en la que se enmarca el Espacio Europeo de la Educación Superior requiere que las universidades europeas tengan un sentido cultural unitario. Sin embargo, el conocimiento de los estudiantes que aporta este estudio pone de manifiesto que no existe esa realidad cultural como tal, por lo que se hace necesario conocer cuáles son las causas de esa situación y sus consecuencias. En este sentido, se aborda la percepción de doscientos sesenta y siete estudiantes de cuarto curso del título de maestro de Educación Primaria sobre la formación didáctica de sus profesores, así como la orientación que reciben y el nivel de compromiso que tienen con el alumnado, mediante un estudio descriptivo-inferencial, con pruebas no paramétricas. El estudio descriptivo utilizó medias, medianas y desviación típica; el estudio inferencial se llevó a cabo mediante

1. Miguel Pérez Ferra es miembro de la RED DIDIPD. 
CRISTO ESPARCIA JIMÉNEZ Y MIGUEL PÉREZ FERRA

VALORACIÓN DE LA FORMACIÓN RECIBIDA POR ESTUDIANTES DEL TÍTULO DE GRADO DE MAESTRO EN EDUCACIÓN PRIMARIA

pruebas no paramétricas: U de Mann Whitney y $\mathrm{H}$ de Kruskal-Wallis. Los resultados indican que hay buena preparación didáctica del profesorado, aunque se evidencia que suele ser en métodos y procedimientos utilizados en la Universidad con anterioridad al EEES, considerando los estudiantes que el profesorado no está preparado en métodos más adecuados para el trabajo autónomo, trabajo en equipo o generación de conocimiento, que suponen un mayor protagonismo del profesorado. Asimismo, consideran los estudiantes que no reciben orientación de sus profesores y perciben falta de compromiso respecto a la formación. Se atisba una tendencia a reducir la profesionalización a mero conocimiento didáctico y un compromiso personal, más vinculado a las vanguardias ideológicas, que a la realidad ética que define las relaciones interpersonales. Se observa, pues, un sentido en la formación de carácter exclusivamente tecnológico, que no técnico, vinculado a una percepción neopositivista, que orienta a la persona exclusivamente a ser competitiva.

Palabras clave: estudiantes de Magisterio; preparación didáctica del profesorado; orientación; compromiso; estudiantes; EEES.

SUMMARY: The knowledge society and globalization in which the European Higher Education Area is framed requires that European universities have a unitary cultural sense. However, the knowledge of the students contributed by this study shows that there is no such cultural reality, so it is necessary to know the causes of this situation and its consequences. In this sense, the perception of two hundred and sixty-seven fourth-grade students of the Primary Education degree on the didactic training of their teachers, as well as the orientation the teachers receive and the level of commitment they have with the students, is addressed through a descriptive inferential study, with non-parametric tests. The descriptive study used means, medians and standard deviations; the inferential study was carried out by non-parametric tests: Mann-Whitney U and Kruskal-Wallis H. The results indicate that there is good teaching preparation by teachers, although it is evident that this preparation is usually by methods and procedures that were used in the University prior to the EHEA, considering students are not prepared in more appropriate methods for autonomous work, teamwork or knowledge generation, which means a greater role for teachers. Also, students believe that they do not receive guidance from their teachers and perceive a lack of commitment to training. There is a tendency to reduce professionalization to mere didactic knowledge and a personal commitment, linked more to an ideological vanguard than to the ethical reality that defines interpersonal relationships. Therefore, there is a sense in the form of an exclusively technological nature, linked to a neo-positivist perception which is not technical and guides the person exclusively to be competitive.

Key words:: teaching students; didactic preparation of teachers; guidance; commitment; students; EHEA. 
Si no nos planteamos la aplicación del Espacio Europeo de Educación Superior (en adelante EEES) como un problema que reclama un cambio de cultura, como una oportunidad para hacerlo, lo que ocurrirá es que se convertirá en una nueva fórmula burocrática de presentar los programas de otra manera (más engorrosa, por cierto), de enunciar cosas que no se harán, si es que llegan a ser comprendidas (Gimeno, 2004, 4).

\section{INTRODUCCIÓN Y ESTADO ACTUAL DE LA CUESTIÓN}

La reflexión precedente, aunque lejana en el tiempo, es de plena actualidad, ya que las evidencias que proporcionan el decurso del tiempo ratifican la reflexión referenciada. No se ha avanzado lo suficiente en cómo el profesorado universitario que imparte docencia en los títulos de maestro debe organizar su práctica docente ante un nuevo modo de concebir la Universidad y, en lo que es también de vital importancia, en la necesidad de analizar si aborda solo aspectos instructivos en su quehacer o si ejercita también su función orientadora.

Estas carencias formativas no siempre han sido adecuadamente abordadas por la Universidad (Buenestado-Fernández, 2019); en unas ocasiones, porque el perfil de las guías docentes ha conducido a una ceremonia de la confusión, de carácter epistemológico; en otras, porque el profesorado no ha recibido una formación adicional bien articulada respecto a la interacción entre conocimientos didáctico-curriculares y su armonización con el proceso de Integración del Sistema Universitario Español en el EEEs, en el que: «[...] la extensión y calidad de la educación superior son factores decisivos en el incremento de la calidad de vida de los ciudadanos" (Ministerio de Educación, Cultura y Deporte, 2003: 1).

Actualmente, se está reduciendo la misión de la Universidad a un posicionamiento de las instituciones universitarias en ranking de excelencia investigadora, como referente último de prestigio. Sin obviar su importancia, solo representa el primer paso en la formación. El compromiso del docente universitario va más allá e implica la formación humanística de sus estudiantes, que comporta orientar al alumnado en la construcción de su «integridad» e «integralidad», solo posible desde una realidad cultural integradora, que será la que defina la relación ética entre estudiante y profesor, "Concretada en una disposición de acogida al "otro" (Ortega-Ruiz, 2004: 5).

La formación de los futuros maestros se sitúa en la complementariedad entre responsabilidad, como dimensión de la libertad, y sentido ético, que transciende lo subjetivo e incluye un compromiso social (Llano, 2011). Sin embargo, corroborando la intuición que planteaba Gimeno (2004), más que un cambio cualitativo de cultura, se ha generado una fórmula burocrática más complicada para llevar a cabo los procesos, que, en no pocas ocasiones, genera una ceremonia de la confusión entre el profesorado que, trece años después, Bolívar (2017) describía del siguiente modo: 
CRISTO ESPARCIA JIMÉNEZ Y MIGUEL PÉREZ FERRA

VALORACIÓN DE LA FORMACIÓN RECIBIDA POR ESTUDIANTES DEL TÍTULO DE GRADO DE MAESTRO EN EDUCACIÓN PRIMARIA

[...] entre guías didácticas por competencias y la llamada "Convergencia en el EEES» [...], junto a un contexto de cambios sociales (utilitarismo y relativismo), se ha perdido lo esencial de la formación universitaria: un sentido ético de las relaciones entre profesores y estudiantes, que caracterizaba ser universitario, con una pérdida de sentido de para qué estamos formando (p. 15).

Las reflexiones precedentes urgen a una toma de decisiones, orientada a simplificar los trámites burocráticos y a un proceso de deconstrucción en la formación docente del profesorado universitario, mediante estudios de caso, resolución de problemas, talleres, etc. De modo singular, debe orientarse a la formación del profesorado novel, ya que accede a la función docente, en no pocas ocasiones, sin conocimientos didáctico-curriculares previos, orientando su docencia al aprendizaje específico del contenido de esas materias, cuando en realidad debiera ser a la formación didáctica de los estudiantes sobre cómo enseñar el contenido específico de esas materias (Montero-Mesa, 2018; Tintaya, 2016).

Además de la formación didáctico-curricular inicial, se les debe informar de la misión institucional de su universidad, para que conozcan la centralidad de las necesidades formativas que plantea el nuevo marco europeo. Para ello, es vital que el profesorado novel conozca los rasgos que deben definir su identidad profesional e identificar qué competencias docentes son necesarias para su desarrollo y ejercicio (Pagés, 2014).

La formación adquirida por los estudiantes está muy vinculada a cómo lleve a cabo el profesorado universitario su función en el aula, relación que requiere determinar en qué es necesario formar; conocer por el docente la realidad ontoepistemológica de la materia; la cultura institucional en la que se encuentra inmerso y qué medios son los más adecuados para llevar a cabo el proceso, así como el referente cultural en el que están inmersos (Laudadío y Da Dalt, 2014).

A lo indicado habría que adicionar: conceptualización del conocimiento por los estudiantes, preparación para el trabajo autónomo, cooperación y generación de conocimiento. Aspectos en los que los estudiantes ponen de manifiesto ciertas carencias, indicadores clave para determinar si la institución universitaria sabe cómo gestionar lo nuevo, en este caso el EEEs, o bien le ha llegado de modo sobrevenido (Llano, 2002).

Ciertamente, la formación se centra en la adquisición de competencias y ello requiere saber hacer, saber ser y saber estar, aspectos necesarios para el ejercicio de la profesión docente (Martínez-Clares y Echeverría, 2009), sin obviar la singularidad de cada persona para su ejercicio, que implica apreciación de la realidad esencial y existencial del futuro docente; es decir, la consideración de su identidad profesional docente (Breijo y Mainegra, 2020; Bolívar y Domingo, 2020; Patrón y Chagoyán, 2019).

Dicho lo cual, elegir una orientación tecnológica en la formación de los futuros docentes contribuye a reducir el rol del profesor al de mero tecnólogo y no al de técnico (Mínguez-Vallejos, 2016). Sin obviar que la acción formativa demanda conocimiento de técnicas, no se debe olvidar que son medios y no fines. Hay dos 
razones justificativas de esta realidad: la primera, más mediata, que incide en que la misión de la Universidad rebasa los parámetros político-económicos; la segunda, más inmediata, y es que las finalidades últimas de la formación de maestros no solo son científicas y, por tanto, no pueden ser objeto estricto de una orientación tecnológica (Romero y Mínguez, 2019).

En esta línea argumental, se trata de que el profesorado contribuya a que los estudiantes adquieran la competencia profesional no solamente en el "saber», sino en el "saber ser" y el "saber estar», que implica asumir que el saber va más allá de la información y supone un crecimiento interno que se manifiesta en la capacidad operativa, que se concreta en la valoración de las potencialidades humanas (Vera, Álava y Basurto, 2019).

Las carencias detectadas en la formación técnica del profesorado universitario se deben a que el "Principio de acción" ha sido interpretado desde la Modernidad como movimiento o cambio, que sitúa a la persona como actor y elude su condición de autor (Tell, 2014; Foucault, 1990) de una acción no temática sino operativa (Llano, 1990). Evidencias que han dado lugar a valorar más lo útil, equiparando lo verdadero con lo empíricamente verificable. (Pérez-Ferra y Quijano, 2018; Mínguez-Vallejos, 2010).

En contraposición a este modo de hacer, se suscribe el pensamiento de Deleuze, determinado por la adscripción del "principio de actividad" a la creatividad y originalidad, como antesala de generación de conocimiento, que es lo que determina que el estudiante sea «autor» y no "actor» en su formación (Etchegaray, 2016).

Las reflexiones previas conducen a pensar que en los momentos actuales no hay identidad cultural propia definida nítidamente en no pocas universidades y, como consecuencia, a los medios se les ha otorgado la categoría de fines.

\section{JUSTIFICACIÓN DE LA INVESTIGACIÓN}

Definir la identidad profesional docente del profesorado universitario que forma a futuros maestros supone asociarla a quien «infunde hábitos, valores y competencias transversales por procedimientos no programados" (Jover, 2016: 11), que deberían primar sobre los del experto en contenidos que implementa y programa.

Ante esta nuevo modo de plantear la Universidad que se plantea en el EEES, los nuevos planes de estudio, que se orientan a la adquisición de competencias, demandan una reflexión sobre qué planteamientos metodológicos son los más adecuados en el ámbito del modelo en el que se trabaja, para facilitar una formación que permita a los futuros egresados desenvolverse de modo efectivo en lo personal y en lo profesional (González-Maura, 2007; González-Tirado, 2005).

El trabajo colaborativo es de vital importancia para ejercitarse en el desarrollo de capacidades y destrezas, imprescindibles en los nuevos perfiles de las titulaciones y para un posterior desempeño de la función profesional docente (Cotán, Martínez-Calderrey, García-Lázaro, Gil-Mediavilla y Gallardo-López, 2020; Zermeño y Zúñiga, 2017). 
CRISTO ESPARCIA JIMÉNEZ Y MIGUEL PÉREZ FERRA

VALORACIÓN DE LA FORMACIÓN RECIBIDA POR ESTUDIANTES DEL TÍTULO DE GRADO DE MAESTRO EN EDUCACIÓN PRIMARIA

Pero no es baladí la competencia didáctica de los docentes, que se vincula con preparar a los estudiantes para elaborar conocimiento, a partir de una disposición indagadora basada en procesos inductivos (Márquez, Reinoso, Pérez y Urbano, 2017). Preparación que, siendo necesaria, no es suficiente (Pérez-Ferra, Quijano y Ocaña, 2013) clarifican esta afirmación indicando que:

La finalidad última de enseñar a pensar no se halla en la resolución de cuestiones meramente fenoménicas, ya que en la Universidad lo mensurable debe ser contingente, sino en el logro de la capacidad científica y tecnológica para generar conocimiento y diseminarlo (p. 243).

Los aspectos abordados son de especial interés, tanto porque definen los procesos para aprender, el modo de encauzarlos, la capacidad de trabajar en grupo, así como el trabajo autónomo para llevarlos a cabo. Pero todo lo indicado, con ser parte integrante del compromiso docente del profesorado, no es suficiente, de modo que son necesarias la ayuda y la orientación al estudiante.

La orientación a los futuros egresados se lleva a cabo facilitando al estudiante la familiarización con los procesos didácticos más comunes seguidos en la Universidad: enfoque del aprendizaje de la materia, procedimientos para buscar información, modos de compartirla con compañeros, cómo generar y compartir conocimiento o trabajar en equipo; de ahí la importancia de una acción tutorial innovadora, que podría ser una "herramienta» de primera magnitud para canalizar aprendizajes, orientar procedimientos para adquirirlos y tomar decisiones, aspectos que mejoran la autoestima y el autoconcepto de los estudiantes.

Las argumentaciones precedentes conducen a un espacio común en el que se articula lo técnico-didáctico con la dimensión orientativa, imprescindible para el desarrollo de competencias, que (Pérez-Ferra, Quijano y Ocaña, 2013.) definen como: «La capacidad de movilizar recursos para poder hacer, pero no entendida como mera aplicación, ni como una reconstrucción, sino como un saber hacer con valor añadido, el de la singularidad de cada persona».

Descripción que aborda las dos funciones básicas del profesor universitario: instructiva y orientadora, pues no solo ayuda al estudiante a activar un conjunto de capacidades y conocimientos y hacerlos operativos, sino también, y aquí es donde entra la función orientativa del docente, ayuda a ejercer la actividad según la singularidad de la personalidad del estudiante.

Profundizar en la razón de ser de la tutoría en la Universidad abre vías para fortalecer la capacitación, formación e inicio de un futuro desarrollo profesional e inserción laboral, aspectos en los que el alumnado detectas carencias formativas (Cano-González, 2009). Además, las demandas del EeEs, implícitas en los enfoques de aprendizaje, urgen a definir sistemas de apoyo al estudiante, para que pueda convertirse en verdadero protagonista de su propia formación.

La acción tutorial constituye un recurso para que los estudiantes puedan recibir una formación personalizada, verdadero leitmotiv de la presente justificación, a fin de ayudarles a disipar sus inquietudes y dudas, respecto a su futuro profesional 
o bien a cómo proceder en los procesos formativos que reciben (Cremades, GarcíaGil, Ramírez-Rico y Miraflores, 2016; Rodríguez-Espinar, 2012).

Las argumentaciones precedentes asumen implícitamente que el profesor universitario no tiene una dualidad de funciones, sino una doble dimensión en su quehacer formativo: acción didáctica y ayuda y orientación al estudiante, siendo el vínculo que une esas dos dimensiones el compromiso ético, reflejado en la "Pedagogía de la Alteridad", entendida como acogida "al otro» que, como se ha afirmado líneas atrás, tiene su centralidad en la acción del docente para ayudar al estudiante a desarrollar sus competencias como futuro profesional.

Las reflexiones anteriores dan soporte a la relevancia de la presente investigación, por la necesidad de excelencia en la formación; por el hecho de evidenciar qué se esconde tras pretendidas mejoras, así como por conocer la percepción de los futuros docentes sobre cómo se les está formando. Dicho lo cual, se plantea el siguiente problema de investigación: ¿Qué percepción tienen los estudiantes de cuarto curso del Grado de Educación Primaria respecto a la preparación didáctica de sus profesores, así como a la orientación y compromiso con los estudiantes?

Respecto al mencionado problema de investigación, se han analizado estudios previos, observándose que los estudiantes no han manifestado diferencias de opinión significativas, en función del género, la edad o el tipo de formación previa de acceso a la carrera (Solbes, Fernández-Sánchez, Domínguez-Sales, Cantó y Guisasola, 2018; Achurra y Morentín, 2017; Pagés, 2014; Torra y Esteban, 2012; García-Berro, Colom, Martínes-Solanas, Sallarés y Roca-Martín, 2011), entre otros investigadores.

\section{Diseño DE LA INVESTIGACIÓN}

Se realizó un estudio descriptivo-inferencial de carácter transversal. Para el estudio descriptivo se utilizaron porcentajes, medidas de tendencia central (media y mediana) y de dispersión (desviación estándar). El estudio inferencial se realizó con pruebas no paramétricas en todos los análisis, ya que, para la totalidad de la muestra, la prueba de Kolmogorov-Smirnov puso de manifiesto que no se cumple el criterio de normalidad en la distribución de la curva, $(p=0.03<0.05)$ y la prueba de Levene, en ningún caso, evidencia homogeneidad de varianzas $\left(t_{(48)}=1.002, p\right.$ $=0.023<0.050$ ).

Se realizó un contraste de medias para la variable dicotómica "género", con la U de Mann Whitney y para las variables categóricas con más de dos categorías, "edad de los estudiantes" y "estudios de acceso", se utilizó la H de Kruskal-Wallis. Los análisis se efectuaron con un nivel de significación de $(p<0.05)$, así como un nivel de confianza del $95 \%$, realizándose con el software informático spss, versión 24.0 de IBM. 
CRISTO ESPARCIA JIMÉNEZ Y MIGUEL PÉREZ FERRA

VALORACIÓN DE LA FORMACIÓN RECIBIDA POR ESTUDIANTES DEL TÍTULO DE GRADO DE MAESTRO EN EDUCACIÓN PRIMARIA

\subsection{Objetivos e hipótesis de la investigación}

- Objetivo general:

- Conocer la percepción de los estudiantes de $4 .^{\circ}$ curso del grado de Educación Primaria sobre el nivel de desempeño, respecto a su competencia profesional docente.

- Objetivos específicos:

- Averiguar la percepción de los estudiantes referenciados sobre la calidad de la enseñanza que imparte el profesorado.

- Analizar la valoración de los estudiantes sobre el ejercicio de la acción orientadora de sus profesores.

\section{- Hipótesis de investigación:}

No hay diferencias estadísticamente significativas entre las variables de segmentación "sexo», «intervalo de edad»y «modo de acceso a los estudios de maestro", en relación a las variables dependientes "preparación didáctica del profesorado» y "orientación y compromiso con los estudiantes".

\subsection{Población y muestra}

Se partió de una población de 440 estudiantes, pertenecientes a la Escuela de Magisterio SAFA de Úbeda, institución privada, adscrita a la Universidad de Jaén, así como los estudiantes de la titulación de dicha Universidad, realizándose un muestreo incidental, ya que la participación de los estudiantes fue voluntaria.

Para el tamaño de la muestra se utilizó la fórmula de cálculo para poblaciones finitas «Sample Size Calculator for a Proportion» (absolute margin) (http://www.berrie. dds.nl/calcss.htm). Teniendo en cuenta la población $(\mathrm{N}=440)$, un error muestral de $5.6 \%$, un nivel de confianza del $95 \%$ y la proporción esperada $(\mathrm{p}=5 \%)$, se obtiene un valor de 206 casos para la justificación de la selección muestral. El número de casos válidos asciende a 267, por encima de la muestra mínima que aporta la aplicación de la fórmula referenciada. La muestra quedó definida del siguiente modo, Tabla 1.

\subsection{Instrumento de recogida de información}

Se elaboró una escala tipo Likert con 30 ítems, con 5 opciones de respuesta, que sirvió de enlace entre los objetivos definidos en este estudio y la muestra seleccionada (De Lara y Ballesteros, 2007). La escala se elaboró con cinco opciones de respuesta: "totalmente en desacuerdo» (TD = 1), "en desacuerdo» $(\mathrm{D}=2)$, «moderadamente de acuerdo" ( $\mathrm{MA}=3$ ), "de acuerdo" $(\mathrm{A}=4)$ y "totalmente de acuerdo" $(\mathrm{TA}=5)$. La revisión de la bibliografía para elaborar la escala permitió definir dos dimensiones en la escala, D-1: Preparación didáctica del profesorado; D-2: Orientación a los estudiantes y compromiso docente. 
CRISTO ESPARCIA JIMÉNEZ Y MIGUEL PÉREZ FERRA

VALORACIÓN DE LA FORMACIÓN RECIBIDA POR ESTUDIANTES DEL TÍTULO DE GRADO DE MAESTRO EN EDUCACIÓN PRIMARIA

TABLA 1

Distribución de la muestra atendiendo a variables descriptivas

\begin{tabular}{|c|c|c|c|}
\hline VARIABLE DESCRIPTIVA & CATEGORÍAS DE LA VARIABLE & FRECUENCIA & PorCENTAJE \\
\hline \multirow{2}{*}{ Sexo } & Hombre & 112 & 41.95 \\
\hline & Mujer & 155 & 58.05 \\
\hline \multirow{3}{*}{$\begin{array}{l}\text { Edad de los } \\
\text { estudiantes }\end{array}$} & Entre 20 y 25 años & 228 & 85.39 \\
\hline & Entre 26 y 30 años & 25 & 9.50 \\
\hline & Más de 31 años & 14 & 5.11 \\
\hline \multirow{4}{*}{$\begin{array}{l}\text { Modo de acceso } \\
\text { a la Universidad }\end{array}$} & Bachiller & 208 & 77.90 \\
\hline & Grado superior de FP & 55 & 20.59 \\
\hline & $\begin{array}{l}\text { Prueba de acceso a } \\
\text { mayores de } 25 \text { años }\end{array}$ & 2 & 0.75 \\
\hline & $\begin{array}{l}\text { Título universitario } \\
\text { oficial de grado }\end{array}$ & 2 & 0.75 \\
\hline
\end{tabular}

FUENTE: Elaboración propia.

\subsection{Validez y fiabilidad del instrumento}

La validez de contenido indicó en qué grado la medida representa a cada elemento de la escala (Salazar-Gómez y Tobón-Juárez, 2018). Se validó mediante el juicios de 8 expertos: 4 tutores del prácticum y 4 profesores de la titulación, considerando dos criterios: la acomodación de cada ítem a lo que pretende valorar el rasgo de la escala y la adecuación semántica de los ítems al nivel de los encuestados. Concluido el proceso se redujo el número de ítems de treinta a veintidós.

\section{- Validez de constructo}

Indicó si el rasgo a valorar de la escala mide el rasgo que se pretende (Schönfeld y Mesurado, 2020). El análisis factorial se realizó mediante el procedimiento de componentes principales ( $\mathrm{PrC}$ ) con fines exploratorios. Los factores se obtuvieron según el criterio de raíz latente de Kaiser-Meyer-Olkin. Posteriormente se aplicó la rotación varimax (máxima varianza) con normalización de Kaiser. La prueba de esfericidad de Bartlett confirmó la existencia de factores subyacentes en la matriz de datos, debido al alto nivel de significación obtenido, Tabla 2. 
CRISTO ESPARCIA JIMÉNEZ Y MIGUEL PÉREZ FERRA

VALORACIÓN DE LA FORMACIÓN RECIBIDA POR ESTUDIANTES DEL TÍTULO DE GRADO DE MAESTRO EN EDUCACIÓN PRIMARIA

TABLA 2

Factorización de variables

\begin{tabular}{|l|l|r|}
\hline Medida de adecuación muestras & Kaiser-Meyer-Olkin & 0.890 \\
\hline \multirow{3}{*}{ Prueba de esfericidad de Bartlett } & Chi-cuadrado & 5924.839 \\
\cline { 2 - 3 } & Gl. & 1225 \\
\cline { 2 - 3 } & Sig. & 0.000 \\
\hline
\end{tabular}

Nota: $(1)=* p<0.05$.

El peso mínimo para establecer la correlación entre variables y factores fue de $p \geq 0,6$, de establecer un peso consistente, que garantice las correlaciones entre variables y factores. El análisis definió 2 factores, que integran diecisiete ítems, definitorios del 37,326\% de la varianza total explicada, Tabla 2.

TABLA 3

Solución factorial definitiva, después de la rotación y varianza total explicada

\begin{tabular}{|c|c|c|}
\hline DiMENSIONES & $\begin{array}{c}\text { VARIANZA TOTAL } \\
\text { EXPLICADA Y POR FACTOR }\end{array}$ & $\begin{array}{l}\text { ÍTEMS QUE INTEGRAN } \\
\text { CADA FACTOR }\end{array}$ \\
\hline $\begin{array}{l}\text { F-1: Preparación didáctica } \\
\text { de los docentes }\end{array}$ & $28.318 \%$ & $\begin{array}{r}2,6,8,9,10,11,12,14,16,17,18 \\
21,22,24\end{array}$ \\
\hline $\begin{array}{l}\text { F-2: Orientación y compro- } \\
\text { miso del profesorado } \\
\text { con los estudiantes }\end{array}$ & $9.008 \%$ & $07,20,24,25$ \\
\hline Varianza total explicada & $37.326 \%$ & \\
\hline Número de factores & 2 & \\
\hline
\end{tabular}

Fuente: Elaboración propia.

\section{- Fiabilidad}

Se determinó la estabilidad de la escala mediante un Alpha de Cronbach para la totalidad $(\alpha=0.970)$, que garantizó la obtención de resultados similares en una misma persona, en momentos diferentes y circunstancias análogas (Gómez del Pulgar et al., 2019), resultando un alpha para la totalidad de $\alpha=0.970$. El Alpha de Cronbach para las dos mitades aportó un resultado para los ítems impares de $\alpha=$ 0.935 , mientras que para los ítems pares fue de $\alpha=0.944$, quedando equilibrada la fiabilidad de los ítems pares, respecto a los impares que integran la escala.

Respecto al Alpha de Cronbach, si se suprime cada ítem se eliminó el ítem n. ${ }^{\circ} 7$, ya que aumenta la fiabilidad (ítem $7=0.971>0.970=$ totalidad escala), con relación a la totalidad, quedando integrada la escala por dieciséis ítems. 

DE MAESTRO EN EDUCACIÓN PRIMARIA

\section{Resultados}

\subsection{Estudio descriptivo}

El análisis de ocho de las variables integradas en la dimensión o factor uno: "Preparación didáctica de los docentes», indica que los estudiantes están "de acuerdo" en que el profesorado "permite, preguntar dudas", "domina la materia que enseña», "utiliza adecuadamente los medios audiovisuales", "utiliza variedad de recursos para la enseñanza", "interacciona con el alumnado", "explican con claridad y generan conocimiento", "relación lo explicado con otros ámbitos disciplinares» y "Contextualizan las ideas y conceptos que explican".

Las variables mencionadas presentan un comportamiento que oscila entre[ítem $8(\mathrm{M}=3.75 / \mathrm{Md}=4.00 / \alpha=0.687)$ ítem $37(\mathrm{M}=3.82 / \mathrm{M}=4 / \alpha=0.893)$, correspondientes a las variables «explican con claridad y generan conocimiento" y "permiten preguntar dudas", respectivamente. Casi la generalidad de variables consideradas evidencian mayoría de opiniones por encima de la media. Si consideramos que la desviación estándar se sitúa entre "baja» y "media-baja», con una desviación típica máxima posible de $\alpha=2$, convendremos que hay un nivel de homogeneidad razonablemente alto en las respuestas y con tendencia a "totalmente de acuerdo".

La percepción desciende a "moderadamente de acuerdo" en las otras seis variables que integran la dimensión "resumen y enfatizan los aspectos clave de cada tema", "muestra interés por renovar contenidos y métodos de enseñanza», "acepta críticas constructivas al desarrollo de la asignatura», "diseña actividades que propician el trabajo autónomo", "relaciona los conceptos nuevos con el conocimiento previo del alumnado", "atiende a los estudiantes que tienen problemas en el aprendizaje de la asignatura".

Los ítems considerados en esta segunda fase oscilan entre ítem 18( $\mathrm{M}=3.31$ / $\mathrm{Md}=3.00 / \alpha=0.905)$ ítem $11(\mathrm{M}=3.51 / \mathrm{Md}=3.00 / \alpha=0.752)$. En ambos casos las valoraciones de los estudiantes se sitúan por encima de la media y la dispersión se distribuye entre media y media-baja.

En la segunda dimensión: «Orientación y compromiso con los estudiantes», las opiniones se concretan en torno al "desacuerdo" ya que afirman que los docentes "no atienden a los alumnos en temas puntuales sobre formación continua y orientación de su futuro"; "no asesoran al alumnado sobre cómo mejorar, partiendo de sus avances", o no orientan a los alumnos sobre "cómo mejorar su estudio y llevar a cabo procesos de autoaprendizaje».

Las valoraciones son concluyentes y oscilan entre ítem $20(\mathrm{M}=2.09 / \mathrm{Md}=$ $2.00 / \alpha=1,077)$ ítem $24(M=2.53 / M d=2 / \alpha=0.982)$. La dispersión es media en las opiniones de los estudiantes. La relación entre media y mediana evidencia más opiniones inferiores a la media; por consiguiente, hay más heterogeneidad en las opiniones de los informantes y tendencia a la baja. 
CRISTO ESPARCIA JIMÉNEZ Y MIGUEL PÉREZ FERRA

VALORACIÓN DE LA FORMACIÓN RECIBIDA POR ESTUDIANTES DEL TÍTULO DE GRADO DE MAESTRO EN EDUCACIÓN PRIMARIA

TABLA 4

Porcentajes y medidas de tendencia central y dispersión

\begin{tabular}{|c|c|c|c|c|c|c|c|c|}
\hline \multirow[t]{2}{*}{ VARIABLES (ÍTEMS) } & \multicolumn{5}{|c|}{ PORCENTAJES } & \multicolumn{3}{|c|}{$\begin{array}{l}\text { MEDIDAS DE } \\
\text { TENDENCIA CENTRAL } \\
\text { Y DISPERSIÓN }\end{array}$} \\
\hline & TD & $\mathrm{D}$ & MA & A & TA & $\overline{\mathrm{X}}$ & $\mathrm{MD}$ & $D T$ \\
\hline \multicolumn{9}{|c|}{ F-1: Preparación didáctica de los docentes } \\
\hline $\begin{array}{l}\text { 2. Domina la materia } \\
\text { que enseña. }\end{array}$ & 0.0 & 0.7 & 11.7 & 73.7 & 13.0 & 4.01 & 4 & 0.535 \\
\hline $\begin{array}{l}\text { 6. Diseñan actividades } \\
\text { que propician el } \\
\text { trabajo autónomo. }\end{array}$ & 1.5 & 3.6 & 43.1 & 46.7 & 5.1 & 3.40 & 4 & 0.719 \\
\hline $\begin{array}{l}\text { 8. Explican con claridad y } \\
\text { generan conocimiento. }\end{array}$ & 0.0 & 2.9 & 28.5 & 59.1 & 9.5 & 3.75 & 4 & 0.687 \\
\hline $\begin{array}{l}\text { 9. Contextualizan las ideas } \\
\text { y concepto que explican. }\end{array}$ & 0.0 & 3.6 & 32.1 & 58.4 & 5.8 & 3.66 & 4 & 0.645 \\
\hline $\begin{array}{l}\text { 10. Relación lo explicado } \\
\text { con otros ámbitos } \\
\text { disciplinares. }\end{array}$ & 0.0 & 5.1 & 27.7 & 61.3 & 5.8 & 3.68 & 4 & 0.663 \\
\hline $\begin{array}{l}\text { 11. Resumen y enfatizan los } \\
\text { aspectos clave de cada tema }\end{array}$ & 0.7 & 9.5 & 43.1 & 42.3 & 4.4 & 3.51 & 3 & 0.752 \\
\hline $\begin{array}{l}\text { 12. Relacionan los conceptos } \\
\text { nuevos con el conocimiento } \\
\text { previo del alumnado. }\end{array}$ & 1.5 & 12.4 & 39.4 & 40.1 & 6.6 & 3.38 & 3 & 0.841 \\
\hline $\begin{array}{l}\text { 14. Muestran interés por } \\
\text { renovar contenidos y } \\
\text { métodos de enseñanza. }\end{array}$ & 2.2 & 10.2 & 27.0 & 55.5 & 5.1 & 3.50 & 4 & 0.841 \\
\hline $\begin{array}{l}\text { 16. Interaccionan } \\
\text { con el alumnado. }\end{array}$ & 1.5 & 3.6 & 2.2 & 61.3 & 12.4 & 3.80 & 4 & 0.832 \\
\hline 17. Permite preguntar dudas. & 0.0 & 3.6 & $8-8$ & 66.4 & 21.1 & 4.05 & 4 & 0.668 \\
\hline $\begin{array}{l}\text { 18. Atienden a los } \\
\text { estudiantes que tienen } \\
\text { problemas en el aprendizaje } \\
\text { de la asignatura. }\end{array}$ & 4.4 & 8.8 & 46.0 & 32.8 & 8.0 & 3.31 & 3 & 0.905 \\
\hline $\begin{array}{l}\text { 21. Utiliza adecuadamente } \\
\text { los medios audiovisuales. }\end{array}$ & 0.0 & 5.8 & 21.2 & 50.4 & 22.6 & 3.90 & 4 & 0.816 \\
\hline
\end{tabular}


CRISTO ESPARCIA JIMÉNEZ Y MIGUEL PÉREZ FERRA

VALORACIÓN DE LA FORMACIÓN RECIBIDA POR ESTUDIANTES DEL TÍTULO DE GRADO DE MAESTRO EN EDUCACIÓN PRIMARIA

\begin{tabular}{|c|c|c|c|c|c|c|c|c|}
\hline \multirow[t]{2}{*}{ VARIABLES (ÍTEMS) } & \multicolumn{5}{|c|}{ PORCENTAJES } & \multicolumn{3}{|c|}{$\begin{array}{l}\text { MEDIDAS DE } \\
\text { TENDENCIA CENTRAL } \\
\text { Y DISPERSIÓN }\end{array}$} \\
\hline & TD & $\mathrm{D}$ & MA & A & TA & $\overline{\mathrm{X}}$ & MD & $D T$ \\
\hline $\begin{array}{l}\text { 22. Utilizan variedad de } \\
\text { recursos para la enseñanza. }\end{array}$ & 0.7 & 9.5 & 17.5 & 51.8 & 20.4 & 3.82 & 4 & 0.983 \\
\hline $\begin{array}{l}\text { 28. Aceptan críticas } \\
\text { constructivas al desarrollo } \\
\text { de la asignatura. }\end{array}$ & 0.7 & 12.4 & 19.7 & 58.5 & 8.8 & 3.42 & 4 & 0.841 \\
\hline $\begin{array}{l}\text { Total F-1 Preparación } \\
\text { didáctica de los docentes. }\end{array}$ & & & & & & & & \\
\hline \multicolumn{9}{|c|}{ F-2: Orientación y compromiso del profesorado con los estudiantes } \\
\hline $\begin{array}{l}\text { 20. Atienden al alumnado } \\
\text { sobre cómo mejorar su } \\
\text { estudio y llevar a cabo } \\
\text { procesos de autoaprendizaje. }\end{array}$ & 35.0 & 35.8 & 16,8 & 9.5 & 2.9 & 2.09 & 2 & 1.077 \\
\hline $\begin{array}{l}\text { 24. No atienden a los } \\
\text { alumnos en temas puntuales } \\
\text { sobre formación continua y } \\
\text { orientación de su futuro. }\end{array}$ & 4.4 & 30.4 & 29.9 & 21.2 & 5.1 & 2.53 & 2 & 0.982 \\
\hline $\begin{array}{l}\text { 25. Evalúan las prácticas } \\
\text { realizadas y orientan al } \\
\text { alumnado sobre cómo } \\
\text { mejorar, partiendo } \\
\text { de sus resultados. }\end{array}$ & 1.5 & 42.3 & 37.2 & 13.9 & 5.1 & 2.43 & 2 & 0.886 \\
\hline $\begin{array}{l}\text { Total F-2: Orientación y } \\
\text { compromiso del profesorado } \\
\text { con los estudiantes. }\end{array}$ & & & & & & & & \\
\hline
\end{tabular}

Nota: $(1)={ }^{*} p<0.05$.

(2): $\mathrm{TA}=$ Totalmente de acuerdo, $\mathrm{A}=$ acuerdo, MA=Moderadamente de acuerdo, $\mathrm{D}=\mathrm{Desacuerdo}$, TA=Totalmente en desacuerdo.

\subsection{Estudio inferencial}

- Contraste de hipótesis entre el «sexo», la «preparación didáctica de los docentes» y "orientación y compromiso con los estudiantes"

Se acepta la hipótesis nula $\left(\mathrm{H}_{0}\right)$; por tanto, el género no incide en la opinión de los estudiantes sobre la formación didáctica del profesorado $\left(X^{2}=2275.0000\right.$, $p=0.944>0.05 . \mathrm{M}=3.78)$, indicando la media $(=3.674)$ que la opinión sobre la formación impartida se orienta hacia el «acuerdo». 
TABLA 5

Contraste de hipótesis entre género y preparación didáctica del profesor

\begin{tabular}{|l|c|c|c|c|}
\hline \multicolumn{2}{|c|}{ GÉNERO } & NÚMERO & $\begin{array}{c}\text { RANGO } \\
\text { PROMEDIO }\end{array}$ & $\begin{array}{c}\text { PREPARACIÓN } \\
\text { DIDÁCTICA }\end{array}$ \\
\hline \multirow{2}{*}{ Preparación didáctica } & Hombre & 112 & 69.28 & \\
\cline { 2 - 5 } & Mujer & 155 & 68.80 & \\
\hline U de Mann-Whitney & & & 2275.0000 \\
\hline \multicolumn{2}{|l|}{ Sig. asintótica (bilateral) } & & & 0.995 \\
\hline
\end{tabular}

Fuente: Elaboración propia, ${ }^{*}(\mathrm{p} \leq 0.05)$.

Del mismo modo, se comprueba que el género del estudiante no incide en la percepción de los estudiantes respecto a si el profesorado orienta y tiene compromiso con ellos $\left(X^{2}=2204.000, p=0.700>0.050\right)$, razón por la que se acepta la hipótesis nula $\left(\mathrm{H}_{0}\right)$. La media $(\mathrm{M}=3.071)$ evidencia que no son frecuentes la orientación ni el compromiso con los estudiantes.

TABLA 6

Contraste de hipótesis entre género, orientación y compromiso del profesorado con los estudiantes

\begin{tabular}{|l|c|c|c|c|}
\hline \multicolumn{2}{|c|}{ GÉNERO } & NÚMERO & $\begin{array}{c}\text { RANGO } \\
\text { PROMEDIO }\end{array}$ & $\begin{array}{c}\text { PREPARACIÓN } \\
\text { DIDÁCTICA }\end{array}$ \\
\hline \multirow{2}{*}{ Preparación didáctica } & Hombre & 112 & 70.50 & \\
\cline { 2 - 5 } & Mujer & 155 & 67.90 & \\
\hline U de Mann-Whitney & & & 2204.0000 \\
\hline \multicolumn{2}{|l|}{ Sig. asintótica (bilateral) } & & & 0.700 \\
\hline
\end{tabular}

Fuente: Elaboración propia, ${ }^{*}(\mathrm{p} \leq .05)$

- Contraste de hipótesis entre el «intervalo de edad", las opiniones respecto a la "preparación didáctica» y la "orientación y compromiso con los estudiantes»

Tampoco el intervalo de edad al que pertenecen los estudiantes consultados influye en la percepción que tienen respecto a la preparación didáctica que atribuyen a sus profesores $\left(X^{2}=1.451, p=0.484>0.005\right)$, aceptándose la hipótesis nula $\left(\mathrm{H}_{0}\right)$. 
CRISTO ESPARCIA JIMÉNEZ Y MIGUEL PÉREZ FERRA

VALORACIÓN DE LA FORMACIÓN RECIBIDA POR ESTUDIANTES DEL TÍTULO DE GRADO DE MAESTRO EN EDUCACIÓN PRIMARIA

\section{TABLA 7}

Contraste de hipótesis entre intervalos de edad y preparación didáctica del profesorado

\begin{tabular}{|l|c|c|c|c|}
\hline \multicolumn{2}{|c|}{ INTERVALO DE EDAD } & NÚMERO & $\begin{array}{c}\text { RANGO } \\
\text { PROMEDIO }\end{array}$ & $\begin{array}{c}\text { PREPARACIÓN } \\
\text { DIDÁCTICA }\end{array}$ \\
\hline \multirow{3}{*}{ Preparación didáctica } & $20-25$ & 228 & 70.68 & \\
\cline { 2 - 5 } & $26-30$ & 25 & 61.57 & \\
\cline { 2 - 5 } & 31 o más & 14 & 56.00 & \\
\hline Chi-cuadrado & & & 1.451 \\
\hline \multicolumn{2}{|l|}{ Sig. asintótica (bilateral) } & & & 0.484 \\
\hline
\end{tabular}

Fuente: Elaboración propia, * $(p \leq 0.05)$.

También se acepta la hipótesis nula $\left(\mathrm{H}_{0}\right)$, concluyendo que el intervalo de edad al que pertenecen los estudiantes no define la opinión de los mismos, en relación a la capacidad orientadora y compromiso del profesorado con los estudiantes $\left(X^{2}=0.748, p=0.688>0.05\right)$.

TABLA 8

Contraste de hipótesis entre intervalos de edad y orientación y compromiso del profesorado con los estudiantes.

\begin{tabular}{|l|c|c|c|c|}
\hline \multicolumn{2}{|c|}{ INTERVALO DE EDAD } & NÚMERO & $\begin{array}{c}\text { RANGO } \\
\text { PROMEDIO }\end{array}$ & $\begin{array}{c}\text { ORIENTACIÓN Y } \\
\text { COMPROMISO CON } \\
\text { LOS ESTUDIANTES }\end{array}$ \\
\hline \multirow{3}{*}{$\begin{array}{l}\text { Orientación y compromiso } \\
\text { con los estudiantes }\end{array}$} & $20-25$ & 228 & 69.50 & \\
\cline { 2 - 5 } & $26-30$ & 25 & 61.46 & \\
\cline { 2 - 5 } & 31 o más & 14 & 75.71 & 0.748 \\
\hline \multicolumn{2}{|l|}{ Chi-cuadrado } & & & 0.688 \\
\hline \multicolumn{2}{|l|}{ Sig. asintótica (bilateral) } & & & \\
\hline
\end{tabular}

Fuente: Elaboración propia,*$(p \leq 0.05)$.

\subsection{Contraste de hipótesis entre "el modo de acceso a la Universidad", la "preparación didáctica" y la "orientación y compromiso con los estudiantes"}

Se acepta la hipótesis nula $\left(\mathrm{H}_{0}\right)$ y se concluye que el modo de acceso a la Universidad de los estudiantes no incide en sus opiniones respecto a la preparación didáctica de los docentes $\left(X^{2}=2.959, p=0.398>0.05\right)$. 

DE MAESTRO EN EDUCACIÓN PRIMARIA

TABLA 9

Contraste de hipótesis entre el modo de acceso a la universidad y preparación didáctica del profesorado

\begin{tabular}{|c|c|c|c|c|}
\hline \multicolumn{2}{|c|}{ MODO DE ACCESO A LA UNIVERSIDAD } & NÚMERO & RANGO & PREPARACIÓN \\
\hline \multirow{4}{*}{$\begin{array}{l}\text { Preparación } \\
\text { didáctica }\end{array}$} & Bachiller & 208 & 70.43 & \\
\hline & $\begin{array}{l}\text { Grado Superior } \\
\text { de FP }\end{array}$ & 55 & 66.82 & \\
\hline & $\begin{array}{l}\text { Prueba de acceso a } \\
\text { mayores de } 25 \text { años. }\end{array}$ & 2 & 17.00 & \\
\hline & $\begin{array}{l}\text { Título universitario } \\
\text { oficial de grado }\end{array}$ & 2 & 29.00 & \\
\hline \multicolumn{2}{|l|}{ Chi-cuadrado } & & & 2.959 \\
\hline \multicolumn{2}{|c|}{ Sig. asintótica (bilateral) } & & & 0.398 \\
\hline
\end{tabular}

Fuente: Elaboración propia, ${ }^{*}(p \leq 0.05)$.

Se acepta la hipótesis nula $\left(\mathrm{H}_{0}\right)$ y se concluye que el modo de acceso a la Universidad de los estudiantes no incide en sus opiniones respecto a la valoración de la capacidad orientativa y de compromiso del profesorado con los estudiantes $\left(X^{2}=3.700, p=0.296>.05\right)$.

TABLA 10

Contraste de hipótesis entre el modo de acceso a la Universidad y orientación y compromiso del profesorado con los estudiantes.

\begin{tabular}{|l|l|c|c|c|}
\hline \multicolumn{2}{|c|}{ MODO DE ACCESO A LA UNIVERSIDAD } & NÚMERO & $\begin{array}{c}\text { RANGO } \\
\text { PROMEDIO }\end{array}$ & $\begin{array}{c}\text { PREPARACIÓN } \\
\text { DIDÁCTICA }\end{array}$ \\
\hline \multirow{4}{*}{$\begin{array}{l}\text { Preparación } \\
\text { didáctica }\end{array}$} & Bachiller & 208 & 69.42 & \\
\cline { 2 - 5 } & Grado Superior de FP & 55 & 70.96 & \\
\cline { 2 - 5 } & $\begin{array}{l}\text { Prueba de acceso a } \\
\text { mayores de 25 años. }\end{array}$ & 2 & 36.00 & \\
\cline { 2 - 5 } & $\begin{array}{l}\text { Título universitario } \\
\text { oficial de grado }\end{array}$ & 2 & 2.50 & \\
\hline Chi-cuadrado & Sig. asintótica (bilateral) & & & 3.700 \\
\hline
\end{tabular}

Fuente: Elaboración propia, ${ }^{*}(p \leq 0.05)$. 
CRISTO ESPARCIA JIMÉNEZ Y MIGUEL PÉREZ FERRA

VALORACIÓN DE LA FORMACIÓN RECIBIDA POR ESTUDIANTES DEL TÍTULO DE GRADO DE MAESTRO EN EDUCACIÓN PRIMARIA

\section{CONCLUSIONES Y DISCUSIÓN}

El estudio ha permitido definir un perfil real y contextualizado de la percepción que tienen los estudiantes de cuarto curso del Título de Grado de Maestro en Educación Primaria, en la provincia de Jaén sobre dos grandes dimensiones relativas a su formación; en primer lugar, la preparación didáctica del profesorado, que repercute necesariamente en la formación didáctico-curricular que han recibido y, consiguientemente, en la adquisición de las competencias transversales y específicas propias del grado; en segundo lugar, han valorado la orientación recibida y el compromiso del profesorado con los estudiantes, que incide en la orientación que reciben para su futuro profesional y en las orientaciones recibidas sobre cómo integran el trabajo autónoma con el colaborativo y los métodos activos de aprendizaje.

Las valoraciones de los estudiantes reflejan aportaciones de interés sobre la adaptación del profesorado al EEEs. Atendiendo a la "preparación didáctica de los docentes", los estudiantes valoran muy positivamente, como cualidades de sus profesores, el dominio de la materia que enseñan, su disponibilidad para resolver dudas, explicar con claridad, indicadores significativos, que -aunque responden a aspectos de la enseñanza universitaria tradicional- son indispensables para el aprendizaje (Martín, 2019). También estiman muy positivamente el desempeño de otros indicadores convergentes con el EEEs, tales como utilización adecuada de medios audiovisuales, de medios de enseñanza o interacción con los alumnos (Ramos-Coaguilla, 2017).

La valoración de los estudiantes es menos positiva respecto a ciertos indicadores también vinculados al EEEs. No facilitan las funciones de análisis y síntesis, ya que no suelen resumir ni enfatizan los aspectos claves de cada tema. Tampoco es común personalizar la acción formativa, en cuanto que no suelen atender a los estudiantes que tienen problemas en el aprendizaje de las asignaturas. En este sentido, conviene precisar que la Universidad, vista desde el EEES, solo es posible, percibida desde una realidad cultural integradora, que define la relación ética entre estudiante y profesor (Ortega-Ruiz, 2004).

Estas apreciaciones de los estudiantes conducen a la consideración de que la realidad cultural a la que se alude no se limita a la utilización de unos métodos u otros, sino a suscribir concepciones educativas diferentes a las pretéritas, como la Educación Personalizada, que atienda al alumnado en su singularidad y en los procesos de interculturalidad, en los que incide el EEEs. Manifestación de esa carencia es la no consideración de las opiniones de los estudiantes sobre el desarrollo de las asignaturas (González-Maura y González-Tirados, 2008; González y Wagenaar, 2005). También otorgan valoración discreta al nivel de desempeño docente para adecuar contenidos y métodos a las demandas sociales que urgen a la Universidad (Zermeño y Zúñiga, 2017; León, Felipe, Iglesias y Latas, 2011).

Es destacable que los estudiantes refieran que el profesorado no atiende a la relación de conceptos nuevos con los conocimientos previos, de modo habitual (significatividad lógica). Aspecto de vital importancia para el aprendizaje significativo y poder trabajar posteriormente con métodos inductivos (Carniel, 2018; González-Maura y González-Tirados, 2008; González y Wagenaar, 2005). 
CRISTO ESPARCIA JIMÉNEZ Y MIGUEL PÉREZ FERRA

VALORACIÓN DE LA FORMACIÓN RECIBIDA POR ESTUDIANTES DEL TÍTULO DE GRADO DE MAESTRO EN EDUCACIÓN PRIMARIA

También otorgan valoración discreta al nivel de desempeño docente para adecuar contenidos y métodos a las demandas sociales que urgen a la Universidad (Zermeño y Zúñiga, 2017; León, Felipe, Iglesias y Latas, 2011). Del mismo modo, consideran que no favorecen decididamente el trabajo autónomo de los estudiantes ni los procesos interdisciplinares desde sus respectivas materias (Márquez, Reinoso, Pérez y Urbano, 2017). Los relatos anteriores, que suponen evidencias del análisis descriptivo, ponen de manifiesto que todavía hay carencias relacionadas en la docencia respecto al nuevo modo de concebir la enseñanza y el aprendizaje, basados en el socioconstructivismo.

La opinión del alumnado también es clarificadora respecto a que sus profesores no les orientan sobre cómo mejorar el rendimiento en su estudio o llevar a cabo acciones de autoaprendizaje. Constatan que no se atiende a los estudiantes en aspectos relativos a la formación continua y orientación en su futuro profesional, ni en cómo mejorar sus prácticas, en función de las carencias observadas (Gómez-Hurtado, Carrasco-Macías y García-Rodríguez, 2017; Herrera-González, 2010), aspecto básico para desarrollar adecuadamente su competencia profesional.

Las evidencias recogidas en los párrafos anteriores vienen corroboradas por el hecho de que no hay diferencias estadísticamente significativas en las opiniones de los estudiantes consultados, respecto a las estimaciones, tanto positivas como negativas, de las dimensiones valoradas; así como por el hecho de que la dispersión oscile entre baja y media; por tanto, con un nivel razonable de homogeneidad.

Los resultados alcanzados alertan de la necesidad de tomar conciencia sobre cómo se está materializando la aplicación del EEes. Que no es solo una cuestión personal de disposición positiva del profesorado, sino también, y sobre todo, de cómo están gestionando este proceso las universidades y las respectivas administraciones educativas, cuya gran carencia es que no hay cultura institucional y, como consecuencia, los medios para materializarla se convierten en fines.

Las conclusiones evidencian que hay un dominio adecuado sobre el desarrollo de algunos procesos didáctico-curriculares, si bien el desempeño de aquellos otros que demanda el EEEs es poco habitual. No se orienta al estudiante ni hay compromiso del docente sobre ello. Llama poderosamente la atención que los profesores interaccionen con los estudiantes, pero no se consideren sus percepciones sobre el desarrollo de las clases.

Asimismo, se resuelven dudas, pero no se adoptan acciones preventivas que atiendan a quienes tienen dificultades de aprendizaje, ni se apuesta por el desarrollo formativo desde la detección de errores en las prácticas, aspectos muy mejorables, articulando procesos mediante una apuesta firme por el aprendizaje significativo, tanto lógico como psicológico.

Otra paradoja que surge del análisis de resultados es que se considere a los docentes capacitados para utilizar medios audiovisuales; para emplear adecuadamente diferentes medios de enseñanza, pero se aluda a una preparación discretabaja en la adecuación de contenidos y métodos a las demandas sociales (Zermeño y Zúñiga, 2017). 
Estas percepciones de los estudiantes se contraponen con la afirmación posterior de que no son capaces de contextualizar contenidos y métodos a la realidad social, lo que viene a confirmar la carencia de conocimientos epistemológicos sobre los métodos utilizados. Contradicciones preocupantes, que evidencian la ausencia de una preparación epistemológica práctica en quien forma como en quien es formado, con el consiguiente peligro de pasar de la subjetividad de la acción didáctica al subjetivismo en los procesos formativos (Sierra-Arizmendiarrieta y Pérez-Ferra, 2007).

El bajo nivel de desempeño de algunos indicadores referenciados queda determinado por las carencias detectadas en la falta de compromiso de una parte del profesorado con cierto nivel de experiencia docente, al que no le urgen a adquirir una preparación adecuada al desempeño de sus funciones. Pero también el profesorado novel carece de una formación inicial básica, no porque no esté dispuesto a recibirla, sino porque las relaciones contractuales con las universidades son precarias, carentes de estabilidad y, por consiguiente, sin un compromiso formativo docente.

Aludiendo a lo anterior, es evidente que no se puede exigir al profesorado novel el cumplimiento de estas exigencias, ya que suele disponer de escasa o nula formación didáctico-curricular inicial; con una dedicación docente máxima, que suele impartir hasta 5 asignaturas en titulaciones distintas, con un número de alumnos que sobrepasan en casi el doble lo que indica Bolonia y con plazos de mínimos para adquirir una acreditación, que si no logran cercena su carrera profesional (ValcárcelCases, 2003).

Las reflexiones precedentes parecen indicar que a la Universidad le ha venido el EEEs de modo sobrevenido. Cierto es que la nueva andadura universitaria ha estado jalonada por grandes escollos económicos, pero no lo es menos que no son pocas las instituciones universitarias que no han estado a la altura de la previsión que requieren cambios de esta envergadura.

Ciertamente, algunos de los indicadores enumerados son invariantes que las universidades han de asumir porque no dependen de ellas, sino casi siempre de carencias económicas de la Administración. Sin embargo, la elaboración de las guías docentes; la asignación a los profesores noveles de créditos que puedan conjugar con su formación docente e investigadora; las propuestas formativas, basadas en las demandas reales del profesorado y no en las ofertas de quienes plantean cursos; la realización de talleres o la definición de una propuesta formativa a corto, medio y largo plazo, relativa a aspectos epistemológico-didácticos de las materias, evitarían esta situación de "provisionalidad" que tiende a ser vitalicia y nos aleja cada vez más de la idea original de la Declaración de Bolonia (Buenestado-Fernández, 2019), sobre todo, cuando ya han trascurrido diez años de su implantación en España.

Las reflexiones precedentes conducen a las reflexiones iniciales con las que se abría esta contribución. Parece ser que la premonición de Gimeno (2004) se está cumpliendo, así lo confirma una voz tan autorizada como la de Bolívar (2017). 
CRISTO ESPARCIA JIMÉNEZ Y MIGUEL PÉREZ FERRA

VALORACIÓN DE LA FORMACIÓN RECIBIDA POR ESTUDIANTES DEL TÍTULO DE GRADO DE MAESTRO EN EDUCACIÓN PRIMARIA

\section{REFERENCIAS BIBLIOGRÁFICAS}

Achurra, A. y Morentín, M. (2017). Análisis del modelo del profesorado en formación sobre las salidas didácticas en educación infantil. Enseñanza de las Ciencias, núm. extraordinario, 2041-2046.

Almudena Cotán, A.; Martínez-Valderrey, V.; García Lázaro, I.; Gil-Mediavilla, M. y GallardoLópez, J. A. (2020). El trabajo colaborativo online como herramienta didáctica en Espacios de Enseñanza Superior (EEES). Percepciones de los estudiantes de los Grados en Educación Infantil y Primaria. Revista d'Innovació Docent Universitària, 12, 82-94. http://dx.doi.org/10.1344/RIDU2020.12.9.

Bolívar, A. (2017). Una ética profesional en la formación y relaciones universitarias. (Autor 2). Barcelona: Octaedro.

Bolívar, A. y Domingo, J. (2020). La investigación (auto) biográfica en educación. Barcelona: Octaedro.

Breijo, T. y Débora Mainegra, D. (2020). En torno al desarrollo de la identidad profesional docente en la formación inicial de los profesores. Mendive. Revista de Educación, 18(1), 1-4. http://mendive.upr.edu.cu/index.php/ MendiveUPR/article/view/1891/1359.

Buenestado-Fernández, M. (2019). La formación docente del profesorado universitario. El caso de la universidad de Córdoba (diagnóstico y diseño normativo). (Tesis doctoral). Universidad de Córdoba, Córdoba, España.

Cano-González, R. (2009). Tutoría universitaria y aprendizaje por competencias ¿Cómo lograrlo? Revista Electrónica Interuniversitaria de Formación del Profesorado, 12(1), 1-25. file://C:/Users/Miguel/Downloads/ Dialnet-TutoriaUniversitariaYAprendizaje PorCompetencias-2956810.pdf.

Carniel, J. (2018). A reflection on the ethics of images in a critical pedagogy for the humanities. Pedagogy, Culture and Society, 26(1), 141-155. https://doi.org/101080/146811366.2017.1364784.

Cremades, R.; García Gil, D.; Ramírez-Rico, E. y Miraflores, E. (2016). Acción tutorial en estudiantes de las menciones de educación física y música del grado de maestro en educación primaria. Revista de Investigación Educativa, 34(2), 417-433. http://dx.doi. org/10.6018/rie.34.2.237931.

Etchegaray, R. (2016). Prólogo no filosófico para un libro de filosofía. En J. P. Esperón, R. Etchegaray, M. Chicolino y A. Romano (Coords.), Pensar con Deleuze. Buenos Aires: Editorial Abierta FAIA.

Foucault, M. (1990). ¿Qué es un autor? México: Universidad Autónoma de Tlaxcala.

García-Berro, E.; Colom, X.; Martínes-Solanas, E.; Sallarés, J. y Roca Martín, S. (2011). La encuesta al alumnado en la evaluación de la actividad docente del profesorado. Aula Abierta, 39(3), 3-14. https://dialnet.unirioja.es/servlet/articulo?codigo=3691446.

Gimeno, J. (2004). El Crédito Europeo: un reto para la calidad de la enseñanza en la universidad. En UCUA, Módulo 15 de Materiales del Proyecto de Formación del Profesorado Universitario - Guía III (pp. 520-552). Córdoba: Unidad para la Calidad de las Universidades Andaluzas.

Gómez del Pulgar, M.; Hernández-Iglesias, S.; Crespo, A.; Pérez-Martín, A. M.; GonzálezJurado, M. A. y Beneis, J. V. (2019). Fiabilidad de una escala para la evaluación competencias enfermeras: estudio de concordancia. Educación Médica, 20(4), 221-230. https://doi.org/10.1016/j.edumed.2018.12.002. 
CRISTO ESPARCIA JIMÉNEZ Y MIGUEL PÉREZ FERRA

VALORACIÓN DE LA FORMACIÓN RECIBIDA POR ESTUDIANTES DEL TÍTULO DE GRADO DE MAESTRO EN EDUCACIÓN PRIMARIA

Gómez-Hurtado, I.; Carrasco-Macías, M. J. y García-Rodríguez, M. P. (2016). Metodologías activas para la enseñanza plurilingüe con estudiantes universitarios. Magis. Revista Internacional de Investigación en Educación, 9(18), 173-192. https://doi.org/10.11144/ Javeriana.m9-18.maep. Fecha de consulta: 10/11/2019.

González-Maura, V. y González Tirados, R. M. (2008). Competencias genéricas y formación profesional: un análisis desde la docencia universitaria. Revista Iberoamericana de Educación, 47, 185-209. https://doi.org/10.35362/ rie470710.

González, J. y Wagenaar, R. (Eds.) (2005). Tuning Educational Structures in Europe. PhaseII. Bilbao: Universidad de Deusto.

González-Tirados, R. M. y González-Maura, V. (2007). Diagnóstico de necesidades y estrategias de formación docente en las universidades. Revista Iberoamericana de Educación, 43(6), 1-14. https://rieoei.org/historico/ deloslectores/1889Maura.pdf.

Herrera-González, J. D. (2010). La formación de docentes investigadores: el estatuto científico de la investigación pedagógica. Magis. Revista Internacional de Investigación en Educación, 3(5), 53-62. http://magisinvestigacioneducacion.javeriana.edu.co/.

Jover, G. (2016). Introducción. En A. Sanmartín (Coord.), La educación en España: el horizonte 2020 (pp. 5-15). Madrid: Fundación FAD.

Laudadío, M. J. y Da Dalt, E. (2014). Estudio de los estilos de enseñanza y estilos de aprendizaje en la universidad. Educación y Educadores, 17(3), 483-498. https://doi. org/10.5294/edu.2014.17.3.5.

León, B.; Felipe, E.; Iglesias, D. y Latas, C. (2011). El aprendizaje cooperativo en la formación inicial del profesorado de Educación Secundaria. Revista de Educación, 354, 715-729. http://www. revistaeducacion. educacion.es/re354/re354_28.pdf.

Llano, A. (1990). Subjetividad moderna y acción trascendental. En R. Alvira (Coord.). Razón y libertad: homenaje a Antonio Millán-Puelles (pp. 63-74). Madrid. España: Rialp.

Llano, A. (2011). Responsabilidad y humanismo en la empresa actual. Cuadernos de Empresa y Humanismo, 116, 85-108. https://dadun.unav.edu/bitstream/10171/35641/1/201403C EyH116-5.pdf.

Llano, A. (septiembre de 2002). La Universidad ante lo nuevo. Conferencia de inauguración del curso 2002-03 en la Universidad de Navarra. Pamplona, Navarra, España. https:// dadun.unav.edu/bitstream/ 10171/ 2516/ 1/Memoria\%20acad\%C3\%A9mica\%20200102.pdf.

Márquez, A.; Reinoso, E.; Pérez, M. I. y Urbano, R. (2017). La formación didáctica del profesorado. Teoría y práctica. InterCambios, 4(1), 73-78. https://ojs.intercambios.cse.udelar. edu.uy/index.php/ic/article/view/116/60.

Martín, A. (2019). El perfil del buen docente universitario según la valoración de alumnos de Magisterio y Psicopedagogía. Perfiles Educativos, 41(164), 61-85. https://doi. org/10.22201/iisue.24486167e.2019.164.58906.

Martínez-Clares, P. y Echeverría, B. (2009). Formación basada en competencias. Revista de Investigación Educativa, 27(1), 125-147.

Mínguez-Vallejos, R. (2010). La escuela hoy en la encrucijada. Hacia otra educación desde la ética de E. Lévinas. Teoría de la Educación, 22(2), 43-61. http://revistas.usal.es/index. php/1130-3743/article/view/8294/8322. Fecha de consulta: 10/11/2019.

Mínguez-Vallejos, R. (2016). Sobre el modo de ser maestro. En I. Carrillo Flores (Coord.), Democracia y Educación en la formación docente (pp. 247-251). Vic: Servicio de Publicaciones de la Universidad de Vic. 
CRISTO ESPARCIA JIMÉNEZ Y MIGUEL PÉREZ FERRA

VALORACIÓN DE LA FORMACIÓN RECIBIDA POR ESTUDIANTES DEL TÍTULO DE GRADO DE MAESTRO EN EDUCACIÓN PRIMARIA

Ministerio de Educación, Cultura y Deporte (2003). La integración del sistema universitario español en el Espacio Europeo de Educación Superior (Documento-Marco). Madrid: Servicio de Publicaciones del Ministerio de Educación, Cultura y Deporte.

Montero-Mesa, L. (2018). Relaciones entre teoría y práctica en la formación inicial. Percepciones de formadores y estudiantes del Grado de Maestro en Educación Primaria. Educatio Siglo XXI, 36(2), 303-330. https://doi.org/10.6018/j/333061.

Ortega-Ruiz, P. (2004). La educación moral como pedagogía de la alteridad. Revista Española de Pedagogía, año LXII, n. ${ }^{\circ}$ 227, 5-30. https://reunir.unir.net/bitstream/handle/123456789/4130/LaEducacionMoralComoPedagogiaDeLaAlteridad. pdf?sequence=1. Fecha de consulta: 10/11/2019.

Pagés, T. (2014). Propuesta de un marco de referencia competencial del profesorado universitario y adecuación de los planes de formación basados en competencias docentes. Proyecto financiado por la REDU (Red Estatal de Docencia Universitaria), 1-212.

Patrón, A. L. y Chagoyan, P. (2020). Los inicios de la identidad profesional en la formación del profesorado de secundaria en Guanajuato, México. Profesorado. Revista de Currículum y Formación del Profesorado, 23(3), 53-70. https://doi.org/10.30827/profesorado. v23i3.9399.

Pérez-Ferra, M. y Quijano, R. (2018). Análisis del discurso de los estudiantes de Magisterio sobre la contribución del practicum al desarrollo de su identidad profesional docente. Educatio Siglo XXI, 36(2), 331-352. https://doi.org/10.6018/j/333091.

Pérez-Ferra, M., Quijano, R y Ocaña, M. T. (2013). El profesorado universitario ante el Espacio Europeo de Educación Superior: Dos años después. Educatio Siglo XXI, 31(2), 235-254. https://revistas.um.es/educatio/article/view/187611.

Ramos-Coaguilla, P. R. (2017). Correlación entre la clase magistral y la toma de apuntes en los estudiantes de maestría de la Universidad San Pedro de Arequipa (Tesis de Maestría). Universidad Nacional de San Agustín de Arequipa.

Rodríguez-Espinar, S. (2012). Manual de tutoría universitaria. Barcelona: Octaedro.

Romero, E. y Mínguez, R. (2019). Presentación: La educación ante los retos de una nueva ciudadanía: implicaciones éticas. Educatio Siglo XX, 37(1), 11-20. https://revistas.um.es/ educatio/article/view/362941/258761.

Salazar-Gómez, E.; Tobón, S. y Juárez, L. G. (2018). Diseño y validación de una rúbrica de evaluación de las competencias digitales desde la socioformación. Revista de Investigación Apuntes Universitarios, 8(3), 24-42. https://doi.org/10.17162/au.v8i3.329.

Schönfeld, F. S. y Mesurado, B. (2020). Adaptación del Cuestionario de Capital Psicológico al ámbito educativo en una muestra argentina. Propósitos y Representaciones, 8(1), 1-13. http://dx.doi.org/10.20511/pyr2020.v8n1.315.

Sierra-Arizmendiarrieta, B y Pérez-Ferra, M. (2007). La comprensión de la relación teoríapráctica: una clave epistemológica de la didáctica. Revista de Educación, 342, 553-576.

Solbes, J.; Fernández-Sánchez, J.; Domínguez-Sales, M. ${ }^{a}$ C.; Cantó, J. y Guisasola, J. (2018). Influencia de la formación y la investigación didáctica del profesorado de ciencias sobre su práctica docente. Enseñanza de las Ciencias, 36(1), 25-44. https://doi.org/10.5565/ $\mathrm{rev} / \mathrm{ensciencias.2355.}$

Tell, M. B. (2014). Después de la Modernidad: reflexión sobre la acción de Dios, la acción del hombre, y cómo la razón se conoce en-acción a sí misma y lo que está encima de ella. Albertus Magnus, 5(1), 11-32. http://dx.doi.org/10.15332/s2011-9771.2014.0001.01.

Tintaya, P. (2016). Enseñanza y desarrollo personal. Revista de Investigación Psicológica, 16, 75-86. http://www.scielo.org.bo/ pdf/rip/ n16/ n16_a05.pdf. 
CRISTO ESPARCIA JIMÉNEZ Y MIGUEL PÉREZ FERRA DE MAESTRO EN EDUCACIÓN PRIMARIA

Torra, I. y Esteban, R. M. (2012). Competencias docentes en la Educación Superior Presentación. REDU - Revista de Docencia Universitaria, Número monográfico, 10(2), 17-20. https://doi.org/10.4995/redu.2012.6095.

Valcárcel-Cases, M. (2003). La Preparación del Profesorado Universitario Español para la Convergencia Europea en Educación Superior. Programa de estudios y análisis destinados a la mejora de la calidad de la enseñanza superior ( $B O E$, Resolución de 13 de mayo de 2003).

Vera, M. B., Álava, S. A. y Basurto, G. (2019). El desempeño profesional: bases de la formación de calidad. Opuntia Brava, 11(1), 224-234. https://doi.org/10.35195/ob.v11i1.716.

Zermeño, E y Zúñiga, S. C. (2017). El trabajo en equipo de estudiantes universitarios y su desempeño en la construcción de prototipo. Anfei-Digital, 7, 1-10. http://anfei.org.mx/ revista/index.php/revista/. 
